\title{
SOME STABLE ALGORITHMS IN RUIN THEORY \\ AND THEIR APPLICATIONS
}

\section{BY}

\author{
DAVID C.M. Dickson
}

The University of Melbourne

Alfredo D. Egídio dos Reis

ISEG, Lisbon

HOWARD R. WATERS

Heriot-Watt University, Edinburgh

\begin{abstract}
In this paper we present a stable recursive algorithm for the calculation of the probability of ultimate ruin in the classical risk model. We also present stable recursive algorithms for the calculation of the joint and marginal distributions of the surplus prior to ruin and the severity of ruin. In addition we present bounds for these distributions.
\end{abstract}

\section{KEYWORDS}

Probability of ruin; Severity of ruin; Surplus prior to ruin; Recursive calculation; Stable algorithm; Compound binomial model.

\section{INTRODUCTION}

In this paper we present recursive algorithms for the (approximate) numerical calculation of various quantities for a classical surplus process. These quantities are the probability of ultimate ruin, the distribution of the severity of ruin, the moments of the severity of ruin, the distribution of the surplus immediately prior to ruin and the joint distribution of the surplus immediately prior to ruin and the severity of ruin. Recursive algorithms for the calculation of some of these quantities have already appeared in the actuarial literature, particularly for the probability of ultimate ruin. However, not all of these algorithms are numerically stable. The stability of recursive algorithms has been discussed by PANJER and WANG (1993) and, in their words, "For unstable recursions, alternative methods of evaluation merit further research". The main purpose of this paper is to present stable ASTIN BULLLETIN, 1995. Vol. 25, No. 2, 153-175 
algorithms. In addition we present bounds and approximations to the (defective) distributions of the severity of ruin and the surplus immediately prior to ruin, and for the (defective) joint distribution of these two quantities.

Our general method for producing algorithms is to approximate the classical surplus process by a discrete process (discrete time and discrete claim amount distribution) and then to derive an algorithm for the appropriate quantity for the discrete model. The discrete model we will be using is an example of a compound binomial model, studies of which have already appeared in the actuarial literature [Gerber (1988), Shiu (1989), Willmot (1992) and Dickson (1994)]. Hence, although in this paper we will regard our algorithms as providing approximations to, for example, the probability of ultimate ruin for a (continuous time) classical surplus process, we could have chosen to regard them as providing exact values for a compound binomial model.

In the next section we introduce the basic continuous time surplus model, the discrete approximation to the basic model and some notation. In Section 3 we discuss the probability of ultimate ruin. In particular, we discuss the stability of some algorithms which have appeared in the actuarial literature, present a new stable algorithm and discuss numerical examples. In Section 4 we consider the calculation of the distribution of the severity of ruin. In Section 5 we use the algorithm presented in Section 4 to derive an algorithm for the calculation of the moments of the severity of ruin. Both the probability of ruin and, perhaps to a lesser extent, the severity of ruin are of obvious interest. Our reasons for considering also the moments of the severity of ruin are that these moments are of some interest in their own right and that these moments can be used to calculate the moments of durations of negative surplus, as shown by Dos ReIs (1993). Finally, in Section 6 we discuss the distribution of the surplus prior to ruin and the joint distribution of the surplus immediately prior to ruin and the severity of ruin.

\section{MODELS AND NOTATION}

Let $\{U(u, t)\}_{t \geq 0}$ be a classical continuous time surplus process, so that

$$
U(u, t)=u+c t-\sum_{i=1}^{N(t)} X_{i}
$$

where :

$u \quad$ is the insurer's initial surplus,

$c$ is the insurer's rate of premium income per unit time,

$N(t) \quad$ is the number of claims in the time interval $(0, t]$ and has a Poisson $(\lambda t)$ distribution, and,

$\left\{X_{i}\right\}_{i=1}^{\infty}$ is a sequence of i.i.d. random variables representing the individual claim amounts.

Throughout this paper we adopt the convention that $\sum_{i=1}^{0}=0$.

We denote by $P(x)$ the distribution function of $X_{i}$. We assume that $P(x)=0$ for $x<0$, so that all claim amounts are non-negative. We assume that the mean of $X_{i}$, 
which we denote $p_{1}$, is finite and that any other moments of $X_{i}$ which we require are also finite. We assume that $c>\lambda p_{1}$.

We define $\theta$ to be such that

$$
c=(1+\theta) \lambda p_{1}
$$

so that $\theta$ is the insurer's premium loading factor.

Without loss of generality, we make the following two assumptions

$$
c=1 \text { and } p_{1}=1
$$

We will refer to the process described above as our "basic process".

We want to produce a discrete approximation to this basic process but before doing so it is convenient to rescale the basic process by multiplying all monetary amounts by some positive number $\beta$ and taking a new time unit to be $\beta^{-1}$ times the original time unit so that the premium income per unit time for the rescaled process is still 1. In all our numerical examples $\beta$ will be 100 .

Now let $\left\{X_{d, i}\right\}_{i=1}^{\infty}$ be a sequence of i.i.d. random variables whose (common) distribution is approximately the same as that of $\beta X_{i}$ and which are distributed on the non-negative integers. We denote the probability function of $X_{d, i}$ by $f(k)$ so that

$$
f(k)=\operatorname{Pr}\left(X_{d, i}=k\right), k=0,1,2, \ldots
$$

Let $N_{d}(t)$ be defined to be $N\left(\beta^{-1} t\right)$ so that $\left\{N_{d}(t)\right\}_{t \geq 0}$ is a Poisson process with parameter $\lambda \beta^{-1}$. Now consider the discrete time surplus process $\left\{U_{d}(u, n)\right\}_{n=0}^{\infty}$ defined as

$$
U_{d}(u, n)=u+n-\sum_{i=1}^{N_{d}(1)} X_{d, i}
$$

so that the premium income per unit time is 1 and the initial surplus is $u$. The implied premium loading factor for this discrete surplus process will be denoted $\theta_{d}$ and is given by the formula

$$
1=\left(1+\theta_{d}\right) \lambda \beta^{-1} E\left[X_{d, i}\right]
$$

Note that if $E\left[X_{d, i}\right]=\beta$ then $\theta_{d}=\theta$. We will always choose $\beta$ and the distribution of $X_{d, i}$ to be such that $\theta_{d}$ is positive. Let $S_{d}$ denote the aggregate claims over the first time period for the discrete model. We will denote by $H_{d}(k)$ and $h_{d}(k)$ the distribution function and probability function, respectively, of $S_{d}$, so that

$$
H_{d}(k)=\sum_{j=0}^{k} h_{d}(j)=\operatorname{Pr}\left(S_{d} \leq k\right)=\operatorname{Pr}\left(\sum_{i=1}^{N_{d}(1)} X_{d, i} \leq k\right) \text { for } k=0,1,2, \ldots
$$

Then it is clear that for any integer $n, U_{d}(\beta u, \beta n)$ will have approximately the same distribution as $U(u, n)$. It should also be clear that by increasing the value of $\beta$ we ought to be able to improve this approximation. 


\section{THE PROBABILITY OF ULTIMATE RUIN}

Let $T$ be the time to ruin for the basic process, starting from initial surplus $u$, so that

$$
T=\left\{\begin{array}{l}
\inf \{t: U(u, t)<0\} \\
\infty \text { if } U(u, t) \geq 0 \text { for all } t>0
\end{array}\right.
$$

The probability of ultimate ruin for the basic process, $\psi(u)$, and its complement, the probability of ultimate survival, $\delta(u)$, are defined as follows

$$
\psi(u)=1-\delta(u)=\operatorname{Pr}(T<\infty)
$$

We are interested in the probability of ruin for our discrete process. However, since we will always take the initial surplus for the discrete process to be an integer we need to define "ruin" carefully. We will use two definitions of ruin for our discrete process, depending on whether or not a surplus of zero, other than at time zero, is regarded as ruin. Accordingly we define

$$
\begin{gathered}
T_{d}=\left\{\begin{array}{l}
\min \left\{n: U_{d}(u, n)<0 \text { for some positive integer } n\right\} \\
\infty \text { if } U_{d}(u, n) \geq 0 \text { for all } n
\end{array}\right. \\
T_{d}^{*}=\left\{\begin{array}{c}
\min \left\{n: U_{d}(u, n) \leq 0 \text { for some positive integer } n\right\} \\
\infty \text { if } U_{d}(u, n)>0 \text { for all } n
\end{array}\right. \\
\psi_{d}(u)=\operatorname{Pr}\left(T_{d}<\infty\right) \\
\psi_{d}^{*}(u)=\operatorname{Pr}\left(T_{d}^{*}<\infty\right)
\end{gathered}
$$

with $\delta_{d}(u)=1-\psi_{d}(u)$ and $\delta_{d}^{*}(u)=1-\psi_{d}^{*}(u)$ denoting the corresponding probabilities of ultimate survival. We need to define the defective probability function of the severity of ruin for the discrete model. For $u=0,1,2, \ldots$ and $y=1$, $2,3, \ldots$, we define

$$
g_{d}(u, y)=\operatorname{Pr}\left(T_{d}<\infty \text { and } U_{d}\left(u, T_{d}\right)=-y\right)
$$

For $u=0,1,2, \ldots$ and $y=0,1,2, \ldots$, we define

$$
g_{d}^{*}(u, y)=\operatorname{Pr}\left(T_{d}^{*}<\infty \text { and } U_{d}\left(u, T_{d}^{*}\right)=-y\right)
$$

It is immediate that

$$
\psi_{d}^{*}=\psi_{d}(u-1) \text { for } u=1,2,3, \ldots
$$

and that

$$
g_{d}^{*}(u, y)=g_{d}(u-1, y+1) \text { for } u=1,2,3, \ldots, \text { and } y=0,1,2, \ldots
$$

It is well known that

$$
\begin{gathered}
\delta_{d}(0)=\theta_{d} /\left[\left(1+\theta_{d}\right) h_{d}(0)\right] \\
\delta_{d}^{*}(0)=\theta_{d} /\left(1+\theta_{d}\right)
\end{gathered}
$$


See, for example, Dickson and Waters (1991, Section 7.1). We need the following formula for $g_{d}(0, y)$

$$
g_{d}(0, y)=\left(1-H_{d}(y)\right) / h_{d}(0) \text { for } y=1,2,3, \ldots
$$

This can be proved by noting first that

$$
g_{d}^{*}(0, y)=1-H_{d}(y)
$$

(this follows from DiCKSON and WATERS [1992, formula (3.5)]) and then conditioning on the aggregate claims in the first time period

$$
g_{d}^{*}(0, y)=h_{d}(0) g_{d}^{*}(1, y)+h_{d}(y+1)
$$

Using (3.6) and (3.2), and rearranging, gives (3.5).

DiCKSON and WATERS (1991, formula (7.2)) presented the following formula for the calculation of $\delta_{d}(u)$ for positive integer values of $u$

$$
\delta_{d}(u)=\frac{1}{h_{d}(0)}\left(\delta_{d}(u-1)-\sum_{i=1}^{u} h_{d}(i) \delta_{d}(u-i)\right)
$$

This formula can be used recursively starting from formula (3.3). We can then use (3.1) to calculate $\delta *(u)$, with $\delta *(0)$ given by (3.4).

In the context of a compound binomial model, this formula has been put forward by Gerber (1988, formulae (6) and (7)), WilLmot (1993, see the remark following formula (3.3)) and DiCKSON (1994, formulae (5.1) and (5.2)). Unfortunately, the recursive scheme based on this formula is not stable. See Dickson and WATERS (1991, Sections 7.2 and 7.3) and PANJer and WANG (1993, Section 11.5).

As an alternative to formula (3.7) we propose the following formula:

$$
\delta_{d}(u)=\delta_{d}(0)+\sum_{k=1}^{u} g_{d}(0, k) \delta_{d}(u-k)
$$

Formula (3.8) can be used to calculate $\delta_{d}(u)$ recursively for $u=1,2,3, \ldots$, starting from (3.3), and using (3.5).

The derivation of (3.8) is elementary. Starting from surplus $u$, ruin does not occur if either the surplus never falls below $u\left(\delta_{d}(0)\right)$ or falls below $u$ for the first time to $u-k$, where $k=1,2, \ldots, u,\left(g_{d}(0, k)\right)$ but ruin does not occur subsequently from this new level $\left(\delta_{d}(u-k)\right)$. The important feature of (3.8) is that it is stable. In fact, Theorem 7 of PANJER and WANG (1993) shows that it is, in their terminology, strongly stable.

By choosing a distribution for $X_{d, i}$ that is, in some sense, a good approximation to that of $\beta X_{j}$ we can use (3.8) to provide a good approximation to $\delta(u)$. For reasons explained by DiCKSON and WATERs (1991, Section 8$), \delta_{d}^{*}(\beta u)$ is usually a better approximation to $\delta(u)$ than is $\delta_{d}(\beta u)$. However, we can also use the discrete model to provide upper and lower bounds for $\delta(u)$.

\section{Result 1}

Let $X_{d, i}$ be defined as follows :

$$
X_{d, i}=k \text { if } k-1 \leq \beta X_{i}<k \text { for } k=1,2, \ldots
$$


Then for any $u>0$

$$
\delta_{d}^{*}([\beta u]) \leq \delta(u)
$$

where $[\beta u]$ is the integer part of $\beta u$.

\section{Proof}

Suppose ruin occurs for the basic process at time $t$, where $(n-1) / \beta<t \leq n / \beta$, for some positive integer $n$. Then for the basic process

$$
u+n / \beta-\sum_{i=1}^{N(n / \beta)} X_{i}<1 / \beta
$$

Hence

$$
\beta u+n-\beta\left(X_{1}+X_{2}+\ldots+X_{N(n / \beta)}\right)<1
$$

Hence for the discrete process

$$
[\beta n]+n-\left(X_{d, 1}+X_{d, 2}+\ldots+X_{d, N_{d}(n)}\right)<1
$$

and so $U_{d}([\beta u], n) \leq 0$. Hence

$$
\psi_{\vec{d}}^{*}([\beta u]) \geq \psi(u)
$$

and the result follows.

\section{Result 2}

Let $X_{d, i}$ be defined as follows

$$
\begin{aligned}
X_{d, i} & =k-1 \text { if } k-1 \leq \beta X_{i}<k \text { for } k=1,2, \ldots, K \\
& =K \text { if } \beta X_{i} \geq K
\end{aligned}
$$

for some positive integer $K$, which could be $\infty$. Then for any $u>0$

$$
\delta * \frac{*}{d}(\{\beta u\}) \geq \delta(u)
$$

where $\{\beta u\}$ is the least integer greater than or equal to $\beta u$.

\section{Proof}

Suppose ruin occurs for the discrete process at time $n$, regarding hitting zero as ruin, starting from initial surplus $\{\beta u\}$. Then:

$$
\{\beta u\}+n-\left(X_{d, 1}+X_{d, 2}+\ldots+X_{d, N_{d}(n)}\right) \leq 0
$$

Hence

$$
\beta u+n-\beta\left(X_{1}+X_{2}+\ldots+X_{N(n / \beta)}\right) \leq 0
$$

Hence

$$
u+n / \beta-\left(X_{1}+X_{2}+\ldots+X_{N(n / \beta)}\right) \leq 0
$$


and so the basic process is ruined at or before time $n / \beta$ starting from initial surplus $u$. Hence

$$
\psi *(\{\beta u\}) \leq \psi(u)
$$

and the result follows.

\section{Comment}

The use of the recursive scheme based on formula (3.8) requires knowledge of the premium loading factor for the discrete model, $\theta_{d}$. This is equivalent to knowing $E\left[X_{d, i}\right]$. When applying Result 2 it may be possible to calculate $E\left[X_{d, i}\right]$ for $K=\infty$, i.e. it may be possible to sum the appropriate infinite series. If not, $K$ can be chosen to be suitably large, but still finite, and $E\left[X_{d i}\right]$ can be calculated by direct summation. The calculation of $E\left[X_{d, i}\right]$, and hence of $\theta_{d}$, for the lower bound in Result 1 may not be quite as simple. If the appropriate infinite series cannot be summed, we can use the fact that for the discrete model

$$
E\left[X_{d, i}\right] \leq 1+\beta E\left[X_{i}\right]=1+\beta
$$

and hence

$$
\theta_{d} \geq \frac{\beta \theta-1}{1+\beta}
$$

and hence

$$
\delta_{d}(0) \geq 1-\frac{1+\beta}{\beta(1+\theta) h_{d}(0)}
$$

Now note that since $g_{d}(0, k)$ is known for all $k$, the values of $\delta_{d}(u)$, for positive integer values of $u$ are all proportional to $\delta_{d}(0)$. Hence, using the right hand side of (3.9) as an approximation to $\delta_{d}(0)$ in formula (3.8) will produce approximations to $\delta_{d}([\beta u])$ which are lower bounds (and which are lower than the correct values of $\delta_{d}([\beta u])$ by the same factor for all $\left.u\right)$ and hence lower bounds for $\delta(u)$.

\subsection{Examples}

In the numerical examples at the end of this section we will compare numerical results produced by formulae (3.3) and (3.8) (and the relationship between $\delta_{d}(u)$ and $\delta_{d}^{*}(u)$ ) with those produced by a different recursive algorithm. This alternative algorithm is called "Method 1" by DUFRESNE and GERBER (1989) and attributed by them to Goovaerts and De Vylder (1984) and Panjer (1986). "Method 1" is a stable recursive scheme since it is based on Panjer's recursion for a compound geometric distribution, which PANJER and WANG (1993, Section 9) show to be stable. It also has the advantage that it produces upper and lower bounds for $\psi(u)$. It requires an interval of discretisation to be chosen. In our examples we will take this to be the unit interval for the rescaled basic process, which is equivalent to an interval of length $\beta^{-1}$ for the basic process. Recall that $\beta=100$ in all our examples. 


\subsubsection{Example 1}

We assume that individual claim amounts for the basic process have an exponential distribution (with mean 1) and that $\theta=0.1$. In this case we can calculate the exact value of $\delta(u)$, which is given by

$$
\delta(u)=1-\frac{1}{1+\theta} \exp \left\{-\frac{\theta u}{1+\theta}\right\}
$$

TABLE 1

See Example 1, Section 3, for details

\begin{tabular}{rrrrrrrr}
\hline \multicolumn{1}{r}{$u$} & $(1)$ & $(2)$ & $(3)$ & $(4)$ & $(5)$ & $(6)$ & $(7)$ \\
\hline 0 & 0.08636 & 0.09091 & 0.09545 & 0.0000 & 0.09091 & 0.09091 & 0.0000 \\
2 & 0.23128 & 0.24204 & 0.25264 & -0.0006 & 0.24142 & 0.24267 & 0.0006 \\
4 & 0.35321 & 0.36805 & 0.38251 & -0.0006 & 0.36701 & 0.36910 & 0.0005 \\
6 & 0.45580 & 0.47311 & 0.48982 & -0.0005 & 0.47181 & 0.47442 & 0.0004 \\
8 & 0.54212 & 0.56070 & 0.57848 & -0.0005 & 0.55925 & 0.56216 & 0.0003 \\
10 & 0.61475 & 0.63373 & 0.65173 & -0.0004 & 0.63222 & 0.63525 & 0.0002 \\
20 & 0.83756 & 0.85243 & 0.86591 & -0.0003 & 0.85121 & 0.85365 & -0.0002 \\
40 & 0.97112 & 0.97605 & 0.98012 & -0.0001 & 0.97565 & 0.97644 & -0.0002 \\
60 & 0.99486 & 0.99611 & 0.99705 & 0.0000 & 0.99601 & 0.99621 & -0.0001 \\
80 & 0.99909 & 0.99937 & 0.99956 & 0.0000 & 0.99935 & 0.99939 & 0.0000 \\
100 & 0.99984 & 0.99990 & 0.99994 & 0.0000 & 0.99989 & 0.99990 & 0.0000 \\
& & & & & & & \\
\hline
\end{tabular}

The columns of Table 1 show for the values of $u$ indicated:

(I) A lower bound for $\delta(u)$ calculated as in Result I. In this example it is easy to show that the premium loading factor for the discrete process, $\theta_{d}$, is $(1+\theta) \beta\left(1-e^{-0.01}\right)-1=0.094518$.

(2) An approximation to $\delta(u)$ based on formula (3.8). The discretisation of the rescaled individual claim amounts for this approximation uses the method of DE Vylder and GoOvaERTS (1988). This method preserves the mean of the distribution so that $\theta_{d}=\theta=0.1$.

(3) An upper bound for $\delta(u)$ calculated as in Result 2 with $K=\infty$. The value of $\theta_{d}$ can be shown to be $(1+\theta) \beta\left(e^{0.01}-1\right)-1=0.105518$.

(4) The relative percentage difference between the approximation in (2) and the correct value for $\delta(u)$, i.e. $100 \times$ (approximation-correct value)/correct value.

(5) A lower bound for $\delta(u)$ calculated using "Method 1 ".

(6) An upper bound for $\delta(u)$ calculated using "Method 1".

(7) The relative percentage difference between the average of the values in (5) and (6) and the correct value for $\delta(u)$.

\subsubsection{Example 2}

Now assume individual claim amounts have a Pareto $(2,1)$ distribution (so that its mean is 1). 
TABLE 2

Sfe Example 2, Section 3, for deTalls

\begin{tabular}{rcccccc}
\hline \hline$u$ & $(1)$ & $(2)$ & $(3)$ & $(4)$ & $(5)$ & $(6)$ \\
\hline 0 & 0.08182 & 0.09091 & 0.09803 & 0.09091 & 0.09091 & 0.09091 \\
2 & 0.17874 & 0.18977 & 0.20337 & 0.18952 & 0.19003 & 0.18978 \\
4 & 0.22726 & 0.25024 & 0.26744 & 0.24992 & 0.25057 & 0.25024 \\
6 & 0.27107 & 0.29785 & 0.31767 & 0.29750 & 0.29821 & 0.29785 \\
8 & 0.30810 & 0.33795 & 0.35983 & 0.33758 & 0.33833 & 0.33796 \\
10 & 0.34045 & 0.37287 & 0.39642 & 0.37249 & 0.37325 & 0.37287 \\
20 & 0.46090 & 0.50186 & 0.53055 & 0.50148 & 0.50224 & 0.50186 \\
40 & 0.60339 & 0.65211 & 0.68446 & 0.65179 & 0.65242 & 0.65211 \\
60 & 0.68750 & 0.73935 & 0.77244 & 0.73911 & 0.73960 & 0.73935 \\
80 & 0.74276 & 0.79598 & 0.82888 & 0.79579 & 0.79617 & 0.79598 \\
100 & 0.78135 & 0.83514 & 0.86755 & 0.83499 & 0.83529 & 0.83514 \\
\hline
\end{tabular}

The columns of Table 2 show for the values of $u$ indicated:

(1) A lower bound for $\delta(u)$ calculated as in Result 1. The value of $\theta_{d}$ has been bounded below as described in the Comment following Result 2, so that its value has been taken to be 0.089109 .

(2) An approximation to $\delta(u)$ based on formula (3.8). The discretisation of the rescaled individual claim amounts for this approximation uses the method of DE VYLDER and Goovaerts (1988), so that $\theta_{d}=\theta=0.1$.

(3) An upper bound for $\delta(u)$ calculated as in Result 2 with $K=35,000$. The value of $\theta_{d}$ can be shown to be 0.108683 .

(4) A lower bound for $\delta(u)$ calculated using "Method 1".

(5) An upper bound for $\delta(u)$ calculated using "Method 1 ".

(6) The average of the values in (4) and (5).

\subsubsection{Comments on Examples 1 and 2}

From Table 1 it can be seen that the numerical results provided by (3.8) and by "Method 1" are very accurate-recall that columns (4) and (7) give the percentage relative errors - at least for exponential claim amounts. For Pareto individual claim amounts we cannot check the accuracy of the two methods, since the exact values are not known, but we can see from Table 2 that the two methods give remarkably similar answers, agreeing to 4 decimal places in all cases and 5 in most. The bounds produced by "Method 1" are closer than those produced by Results 1 and 2 .

\section{THE PROBABILITY AND SEVERITY OF RUIN}

In this section we present a stable recursive algorithm for the approximate numerical calculation of the probability and severity of ruin for our basic process. Using a different approach, we also derive lower and upper bounds for this quantity.

Let $G(u, y)$ denote the probability that ruin occurs for our basic process, given initial surplus $u$, and that the deficit at the time of ruin is less than $y$, so that for 
$u \geq 0$ and $y>0$

$$
G(u, y)=\operatorname{Pr}(T<\infty \text { and } U(u, T)>-y)
$$

Using the discrete approximation to our basic process, an approximation to $G(u, y)$ is $G_{d}^{*}(\beta u, \beta y)$ where

$$
G_{d}^{*}(u, y)=\operatorname{Pr}\left(T_{d}^{*}<\infty \quad \text { and } \quad U_{d}\left(u, T_{d}^{*}\right)>-y\right)
$$

DiCKSON and WATERS (1992, Section 3) presented the following formula for the calculation of $G_{d}^{*}(u, y)$ for $u=0,1,2, \ldots$ and $y=1,2,3, \ldots$

$$
G_{d}^{*}(u+1, y)=\frac{1}{h_{d}(0)}\left(G_{d}^{*}(u, y)-\sum_{j=1}^{u} h_{d}(j) G_{d}^{*}(u+1-j, y)+H_{d}(u)-H_{d}(u+y)\right)
$$

This formula allows recursive calculation of $G_{d}^{*}(u, y)$ starting from

$$
G_{\tilde{d}}^{*}(0, y)=\sum_{j=0}^{y-1}\left(1-H_{d}(j)\right)
$$

Although this algorithm provides very good approximations for moderate values of $u$, it is unstable. An alternative approach to calculating $G_{d}^{*}(u, y)$ is as follows. Define, for $u=0,1,2, \ldots$ and $y=1,2,3, \ldots$,

$$
G_{d}(u, y)=\operatorname{Pr}\left(T_{d}<\infty \text { and } U_{d}\left(u, T_{d}\right) \geq-y\right)
$$

Then for $u=0,1,2, \ldots$

$$
G_{d}(u, y)=\sum_{j=1}^{y} g_{d}(u, j)=\sum_{j=1}^{y} g_{d}^{*}(u+1, j-1)=G_{d}^{*}(u+1, y)
$$

We can calculate $G_{d}(u, y)$ for $u=1,2,3, \ldots$ and $y=1,2,3, \ldots$ from the following formula :

$$
G_{d}(u, y)=G_{d}(0, u+y)-G_{d}(0, u)+\sum_{k=1}^{u} g_{d}(0, k) G_{d}(u-k, y)
$$

This formula follows by considering the level of the surplus process on the first occasion that the surplus falls below its initial level (if this ever occurs). We can calculate $G_{d}(0, y)$ in a recursive manner from (3.5) and hence can also calculate $G_{d}(u, y)$ recursively. Once again, by Theorem 7 of PANJER and WANG (1993), this is a stable recursive algorithm.

We will give an example to illustrate the use of this algorithm at the end of this section. Before doing so we show how to derive lower and upper bounds for $G(u, y)$. The method does not involve the discrete approximation to the basic process. For the remainder of this section we will make the additional assumption that $P(x)$ is absolutely continuous and we will denote its density function by $p(x)$.

Let $g(u, y)$ denote the derivative of $G(u, y)$ with respect to $y$. It is well known that

$$
g(0, y)=\frac{\lambda}{c}(1-P(y))
$$


(see, for example, Gerber et al. (1987)). We can write

$$
g(u, y)=\frac{1}{\delta(0)}\left(\frac{\lambda}{c} \int_{0}^{u} p(y+z) \psi(u-z) d z+g(0, u+y)-\psi(u) g(0, y)\right)
$$

(see PANJER and Willmot (1992) or DiCKSON and DOS REIS (1994)) and it follows by integrating (4.4) over $y$ that

$$
\begin{aligned}
G(u, y)= & \frac{1}{\delta(0)}\left(\int_{0}^{u} \psi(u-z)[g(0, z)-g(0, z+y)] d z\right. \\
& +G(0, u+y)-G(0, u)-\psi(u) G(0, y)) \\
= & \frac{1}{\partial(0)}\left(\sum_{r=0}^{u-1} \int_{r}^{r+1} \psi(u-z)[g(0, z)-g(0, z+y)] d z\right. \\
& +G(0, u+y)-G(0, u)-\psi(u) G(0, y))
\end{aligned}
$$

Now let $\psi^{\prime}(u)$ and $\psi^{h}(u)$ denote lower and upper bounds respectively for $\psi(u)$, calculated, for example, by one of the methods in the previous section.

From (4.5), a lower bound for $G(u, y)$ is $G^{l}(u, y)$ where

$$
\begin{aligned}
G^{\prime}(u, y)= & \frac{1}{\delta(0)}\left(\sum_{r=0}^{u-1} \psi^{\prime}(u-r) \int_{r}^{r+1}[g(0, z)-g(0, z+y)] d z\right. \\
& \left.+G(0, u+y)-G(0, u)-\psi^{h}(u) G(0, y)\right) \\
= & \frac{1}{\delta(0)}\left(\sum_{r=0}^{u-1} \psi^{l}(u-r)[G(0, r+1)-G(0, r)]\right. \\
& -\sum_{r=0}^{u-1} \psi^{l}(u-r)[G(0, r+y+1)-G(0, r+y)] \\
& \left.+G(0, u+y)-G(0, u)-\psi^{h}(u) G(0, y)\right)
\end{aligned}
$$

and an upper bound is $G^{h}(u, y)$ where

$$
\begin{aligned}
G^{h}(u, y)= & \frac{1}{\delta(0)}\left(\sum_{r=0}^{u-1} \psi^{h}(u-r-1)[G(0, r+1)-G(0, r)]\right. \\
& -\sum_{r=0}^{u-1} \psi^{h}(u-r-1)[G(0, r+y+1)-G(0, r+y)] \\
& \left.+G(0, u+y)-G(0, u)-\psi^{l}(u) G(0, y)\right)
\end{aligned}
$$


Since it is always possible to compute $G(0, y)$, either because (4.3) can be integrated in closed form or because we can integrate (4.3) numerically to any degree of accuracy we choose, we can always compute these bounds for $G(u, y)$. In our examples we will calculate bounds for the rescaled basic process, with $\beta=100$.

\subsection{Examples}

\subsubsection{Example 3}

Table 3 shows bounds and exact values for, and approximations to, $G(u, y)$ when the individual claim amount distribution is exponential with mean $1, \theta=0.1$ and the bounds for $\psi(u)$ have been calculated using "Method 1" as described in the previous section. The key to Table 3 is as follows:

(1) gives the value of $G^{l}(u, y)$,

(2) gives the exact value of $G(u, y)$ (see, for example, DiCKSon (1992)),

(3) gives the approximation to $G(u, y)$, calculated from the recursive algorithm,

(4) gives the approximation to $G(u, y)$, calculated by averaging the lower and upper bounds, and

(5) gives the value of $G^{h}(u, y)$.

TABLE 3

See Example 3, Section 4, for detalls

\begin{tabular}{llccc}
\hline \hline & & $y=1$ & $y=3$ & $y=5$ \\
\hline$u=20$ & $(1)$ & 0.077091 & 0.115884 & 0.121134 \\
& $(2)$ & 0.093278 & 0.140217 & 0.146570 \\
& $(3)$ & 0.093034 & 0.140119 & 0.146549 \\
& $(4)$ & 0.093279 & 0.140219 & 0.146572 \\
$u=60$ & $(5)$ & 0.109468 & 0.164554 & 0.172009 \\
& $(1)$ & 0.001179 & 0.001772 & 0.001852 \\
& $(2)$ & 0.002458 & 0.003694 & 0.003862 \\
& $(3)$ & 0.002451 & 0.003692 & 0.003861 \\
& $(4)$ & 0.002458 & 0.003695 & 0.003863 \\
& $(5)$ & 0.003738 & 0.005619 & 0.005874 \\
& $(1)$ & 0.000009 & 0.000013 & 0.000014 \\
& $(2)$ & 0.000065 & 0.000097 & 0.000102 \\
& $(3)$ & 0.000065 & 0.000097 & 0.000102 \\
& $(4)$ & 0.000065 & 0.000097 & 0.000102 \\
& $(5)$ & 0.000121 & 0.000182 & 0.000190 \\
\hline
\end{tabular}

\subsubsection{Example 4}

Table 4 shows bounds for, and approximations to, $G(u, y)$ when the individual claim amount distribution is Pareto $(2,1), \theta=0.1$ and the bounds for $\psi(u)$ have been calculated using "Method 1" as described in the previous section. The key to Table 4 is as follows:

(1) gives the value of $G^{l}(u, y)$,

(2) gives the approximation to $G(u, y)$, calculated from the recursive algorithm, 
(3) gives the approximation to $G(u, y)$, calculated by averaging the lower and upper bounds, and

(4) gives the value of $G^{h}(u, y)$.

TABLE 4

See Example 4, Section 4, for detail.s

\begin{tabular}{lcccc}
\hline \hline & & $y=1$ & $y=5$ & $y=10$ \\
\hline \multirow{2}{*}{$u=20$} & $(1)$ & 0.075914 & 0.204566 & 0.274804 \\
& $(2)$ & 0.079821 & 0.211242 & 0.282126 \\
& $(3)$ & 0.079990 & 0.211347 & 0.282184 \\
& $(4)$ & 0.084065 & 0.218128 & 0.289563 \\
$u=100$ & $(1)$ & 0.011382 & 0.033331 & 0.047841 \\
& $(2)$ & 0.012918 & 0.035929 & 0.050693 \\
& $(3)$ & 0.012945 & 0.035948 & 0.050705 \\
& $(4)$ & 0.014509 & 0.038566 & 0.053569 \\
& $(1)$ & 0.003056 & 0.009230 & 0.013560 \\
& $(2)$ & 0.003593 & 0.010137 & 0.014554 \\
& $(3)$ & 0.003601 & 0.010142 & 0.014558 \\
& $(4)$ & 0.004146 & 0.011054 & 0.015555 \\
\hline
\end{tabular}

\subsubsection{Comments on Examples 3 and 4}

(i) In each example, the two approximations to $G(u, y)$ are close to each other. We can see in Example 3 that for smaller values of $u$, the approximation based on the bounds is slightly superior, but for large values of $u$ both approximations give values very close to the true value.

(ii) The calculation of $G^{l}(u, y)$ and $G^{h}(u, y)$ is not recursive so that separate calculations are required for each combination of $u$ and $y$. The calculation of $G_{d}(u, y)$ using (4.2) is recursive in $u$, and so is more convenient if values are required for several values of $u$.

\section{MOMENTS OF THE SEVERITY OF RUIN}

In this section we are interested in the moments of the severity of ruin for the basic process. For this process, let $Y$ be a defective random variable denoting the severity of ruin. The $k$-th unconditional moment of $Y$ is given by

$$
E\left(Y^{k} \mid u\right)=\int_{0}^{\infty} y^{k} g(u, y) d y
$$

and the conditional moment is found by dividing this quantity by $\psi(u)$.

We can use results from the previous two sections to obtain approximations to these moments.

Let $Y_{d}$ and $Y_{d}^{*}$ denote the deficit at the time of ruin for the discrete process, the distinction being that, for the latter, a surplus of zero, other than at time zero, is 
regarded as ruin. The unconditional moments of these defective random variables are given by

$$
E\left(Y_{d}^{k} \mid u\right)=\sum_{y=1}^{\infty} y^{k} g_{d}(u, y) \text { and } E\left(Y_{d}^{*} \mid u\right)=\sum_{y=0}^{\infty} y^{k} g_{d}^{*}(u, y)
$$

We will approximate $(5.1)$ by $\beta^{-k} E\left(Y_{d}^{* k} \mid \beta u\right)$. We will derive a recursive algorithm for $E\left(Y_{d}^{k} \mid u\right)$ and then use this to calculate $E\left(Y_{d}^{*} \mid u\right)$. In our examples we will consider only the first three moments.

Since $g_{d}^{*}(u, y)=g_{d}(u-1, y+1)$ for $u=1,2,3, \ldots$ and $y=0,1,2, \ldots$, it follows that for $u=1,2,3, \ldots$

$$
\begin{aligned}
& E\left(Y_{d}^{*} \mid u\right)=E\left(Y_{d} \mid u-1\right)-\psi_{d}(u-1) \\
& E\left(Y_{d}^{*} \mid u\right)=E\left(Y_{d}^{2} \mid u-1\right)-2 E\left(Y_{d} \mid u-1\right)+\psi_{d}(u-1) \\
& E\left(Y_{d}^{*} \mid u\right)=E\left(Y_{d}^{3} \mid u-1\right)-3 E\left(Y_{d}^{2} \mid u-1\right)+3 E\left(Y_{d} \mid u-1\right)-\psi_{d}(u-1)
\end{aligned}
$$

For $u=0$ we have

$$
\begin{aligned}
E\left(Y_{d}^{*} \mid 0\right) & =\sum_{y=0}^{\infty} y g_{d}^{*}(0, y) \\
& =\sum_{y=0}^{\infty} y\left(1-H_{d}(y)\right) \\
& =\frac{1}{2}\left(E\left(S_{d}^{2}\right)-E\left(S_{d}\right)\right)
\end{aligned}
$$

Similarly

$$
E\left(Y_{d}^{2} \mid 0\right)=\frac{1}{3} E\left(S_{d}^{3}\right)-\frac{1}{2} E\left(S_{d}^{2}\right)+\frac{1}{6} E\left(S_{d}\right)
$$

and

$$
E\left(Y_{d}^{3} \mid 0\right)=\frac{1}{4} E\left(S_{d}^{4}\right)-\frac{1}{2} E\left(S_{d}^{3}\right)+\frac{1}{4} E\left(S_{d}^{2}\right)
$$

with

$$
E\left(Y_{d}^{k} \mid 0\right)=h_{d}(0)^{-1} E\left(Y_{d}^{* k} \mid 0\right) \text { for } k=1,2,3, \ldots
$$

From (4.2) it is easy to see that for $u=1,2,3, \ldots$ and $y=1,2,3, \ldots$

$$
g_{d}(u, y)=g_{d}(0, u+y)+\sum_{k=1}^{u} g_{d}(0, k) g_{d}(u-k, y)
$$

and so

$$
\begin{aligned}
E\left(Y_{d}^{k} \mid u\right) & =\sum_{y=1}^{\infty} y^{k} g_{d}(0, u+y)+\sum_{y=1}^{\infty} y^{k} \sum_{k=1}^{u} g_{d}(0, k) g_{d}(u-k, y) \\
& =\sum_{k}(u)+\sum_{k=1}^{u} g_{d}(0, k) E\left(Y_{d}^{k} \mid u-k\right)
\end{aligned}
$$


where

$$
\Sigma_{k}(u)=\sum_{y=1}^{\infty} y^{k} g_{d}(0, u+y)
$$

Assuming $\Sigma_{k}(u)$ is known, (5.2) is a stable recursion formula for $E\left(Y_{d}^{k} \mid u\right)$. Note that $\Sigma_{k}(0)=E\left(Y_{d}^{k} \mid 0\right)$, which is known. We can calculate $\Sigma_{k}(u)$ recursively for $u=$ $1,2,3, \ldots$ as follows :

$$
\begin{aligned}
& \Sigma_{1}(u+1)=\Sigma_{1}(u)-\psi_{d}(0)+G_{d}(0, u) \\
& \Sigma_{2}(u+1)=\Sigma_{2}(u)-2 \Sigma_{1}(u+1)-\psi_{d}(0)+G_{d}(0, u) \\
& \Sigma_{3}(u+1)=\Sigma_{3}(u)-3 \Sigma_{2}(u+1)-3 \Sigma_{1}(u+1)-\psi_{d}(0)+G_{d}(0, u)
\end{aligned}
$$

Unfortunately, these recursion formulae are unstable. In our examples we have applied these formulae but have constrained them to satisfy the following inequalities :

$$
\begin{array}{lll}
0 \leq \Sigma_{k}(u+1) \leq \sum_{k}(u) & \text { for } k=1,2,3 & \text { and } u=0,1,2, \ldots \\
\Sigma_{k}(u) \leq \Sigma_{k+1}(u) & \text { for } k=1,2 & \text { and } u=0,1,2, \ldots
\end{array}
$$

\subsection{Examples}

We have used the method of this section to calculate the conditional moments of the severity of ruin in two cases: firstly, when individual claim amounts have an exponential distribution with mean 1 and, secondly, when they have a Pareto $(4,3)$ distribution. Thus, we have calculated

$$
\beta^{-k} E\left(Y^{* k} \mid \beta u\right) / \psi *(\beta u)
$$

and we regard this as an approximation to the conditional moment $E\left(Y^{k} \mid u\right) / \psi(u)$ for the basic process.

The calculation of $E\left(Y_{d}^{*} \mid 0\right)$ requires $E\left(S_{d}^{k+1}\right)$ to be finite. For this reason, we have calculated just the first two conditional moments of the severity of ruin for the Pareto $(4,3)$ distribution. For the exponential distribution, where $E\left(S_{d}^{k+1}\right)$ is finite for all $k$, we have calculated the first three moments.

PANJER and LUTEK (1983) describe a method which may provide a discretisation of the rescaled individual claim amount distribution that preserves the moments of the original distribution. Because we need values of $E\left(S_{d}^{k+1}\right)$ we have adopted this discretisation method for this section only. PANJER and LuTEK (1983) mention the possibility of obtaining negative values for probabilities under this method. In the examples below we used the software Mathematica and specified a high numerical precision for all calculations in the discretisation procedure. In this way we obtained positive values for all probabilities in the discretised distribution.

\subsubsection{Example 5}

When the individual claim amount distribution is exponential, so too is the distribution of the severity of ruin given that ruin occurs. In particular, $E\left(Y^{k} \mid u\right)$ / 
$\psi(u)$ is independent of $u$. Hence, when the individual claim amount is exponential,

$$
\begin{gathered}
E(Y \mid u) / \psi(u)=1 \\
E\left(Y^{2} \mid u\right) / \psi(u)=2 \\
E\left(Y^{3} \mid u\right) / \psi(u)=6
\end{gathered}
$$

The method of this section gives the following results for $\theta=0.1$ :

$$
\begin{aligned}
& \beta^{-1} E\left(Y_{d}^{*} \mid \beta u\right) / \psi_{d}^{*}(\beta u)=\left\{\begin{array}{l}
0.9995 \text { for } u=0,2,3,4, \ldots, 100 \\
0.9996 \text { for } u=1
\end{array}\right. \\
& \beta^{-2} E\left(Y_{d}^{*} \mid \beta u\right) / \psi_{d}^{*}(\beta u)=2.0082 \text { for } u=0,1,2, \ldots, 100 \\
& \beta^{-3} E\left(Y_{d}^{*} \mid \beta u\right) / \psi_{d}^{*}(\beta u)=6.0518 \text { for } u=0,1,2, \ldots, 100
\end{aligned}
$$

In this example it was necessary to apply the constraints described above in the calculation of the functions $\Sigma_{k}(u)$, for $k=1,2,3$.

\subsubsection{Example 6}

Now suppose that the individual claim amounts have a Pareto $(4,3)$ distribution. The method of this section gives the results in Table 5 for $\theta=0.1$.

TABLE 5

See Example 6, Section 5, for details

\begin{tabular}{rcc}
\hline \hline$u$ & $\beta^{-1} E(Y * \mid \beta u) / \psi *(\beta u)$ & $\beta^{-2} E\left(Y *^{2} \mid \beta u\right) / \psi *(\beta u)$ \\
\hline 0 & 1.4995 & 9.0123 \\
40 & 3.7585 & 111.85 \\
120 & 5.8098 & 432.11 \\
160 & 11.670 & $1,868.1$ \\
200 & 27.067 & $7,098.8$ \\
& 52.985 & 18,892 \\
\hline
\end{tabular}

Using formula (4.3) and (5.1) and the fact that $\psi(0)=1 /(1+\theta)$, it is easy to show that $E(Y \mid 0) / \psi(0)=1.5$ and $E\left(Y^{2} \mid 0\right) / \psi(0)=9$ in this example. It is not possible to check the accuracy of the results in this example, other than when $u=0$. It is, however, interesting to note that the conditional moments of $Y^{*}$ increase with $u$.

In this example there was no need to apply the constraints described above in the calculation of the functions $\Sigma_{k}(u)$, for $k=1,2,3$.

\section{DISTRIBUTIONS FOR THE SURPLUS PRIOR TO RUIN}

In this section we present stable recursive algorithms for the approximate numerical calculation of the (defective) distribution of the surplus immediately prior to ruin, and for the (defective) joint distribution of the surplus immediately prior to ruin and the severity of ruin for our basic process. We will also apply the ideas introduced in Section 4 to derive bounds for these distributions. 
Define $U(u, \tilde{T})$ to be the surplus immediately prior to ruin for our basic process and for $u \geq 0$ and $x>0$ define

$$
F(u, x)=\operatorname{Pr}(T<\infty \text { and } U(u, \tilde{T})<x)
$$

so that $F(u, x)$ is the probability that ruin occurs (from initial surplus $u$ ) and that the surplus immediately prior to ruin is less than $x$.

Using the discrete approximation to our basic process, an approximation to $F(u, x)$ is $F_{d}^{*}(\beta u, \beta x)$ where for $u=0,1,2, \ldots$ and $x=1,2,3, \ldots$

$$
F_{d}^{*}(u, x)=\operatorname{Pr}\left(T^{*}<\infty \text { and } U_{d}\left(u, T_{d}^{*}-1\right)<x\right)
$$

DICKSON (1992) presents the following formulae for the calculation of $F_{d}^{*}(u, x)$ for $x=1,2,3, \ldots$ :

$$
F_{d}^{*}(u, x)=\frac{1}{h_{d}(0)}\left(F^{*}(u-1, x)-\sum_{j=u}^{\infty} h_{d}(j)-\sum_{j=1}^{u-1} h_{d}(j) F_{d}^{*}(u-j, x)\right)
$$

for $u=0,1,2, \ldots, x$ and

$$
F_{d}^{*}(u, x)=\frac{1}{h_{d}(0)}\left(F_{d}^{*}(u-1, x)-\sum_{j=1}^{u-1} h_{d}(j) F_{d}^{*}(u-j, x)\right)
$$

for $u=x+1, x+2, x+3, \ldots$. We can use these formulae to calculate $F *(u, x)$ recursively starting from

$$
F_{d} *(0, x)=\sum_{j=0}^{x-1}\left(1-H_{d}(j)\right)
$$

This algorithm provides good approximations for moderate values of $u$ but is unstable. To provide an alternative method of calculating $F *(u, x)$ we require the following definitions. For $u=0,1,2, \ldots$ and $x=1,2,3, \ldots$, define

$$
F_{d}(u, x)=\operatorname{Pr}\left(T_{d}<\infty \text { and } U_{d}\left(u, T_{d}-1\right)<x\right)
$$

and for $u=0,1,2, \ldots, x=0,1,2, \ldots$ and $y=1,2,3, \ldots$, define

$$
f_{d}(u, x, y)=\operatorname{Pr}\left(T_{d}<\infty, U_{d}\left(u, T_{d}\right)=-y \text { and } U_{d}\left(u, T_{d}-1\right)=x\right)
$$

Now for $u=1,2,3, \ldots$ and $x=1,2,3, \ldots$

$$
F_{d}^{*}(u, x)=F_{d}(u-1, x-1)
$$

We can find $F_{d}(0, x)$ by conditioning on the aggregate claim amount in the first time period. We have

$$
F_{d}^{*}(0, x)=h_{d}(0) F_{d}^{*}(1, x)+1-h_{d}(0)
$$


Substituting $(6.1)$ for $F_{d}^{*}(0, x)$ and $F_{d}(0, x-1)$ for $F^{*}(1, x)$, and rearranging leads to

$$
F_{d}(0, x)=\frac{1}{h_{d}(0)} \sum_{j=1}^{x}\left(1-H_{d}(j)\right)
$$

We can calculate $F_{d}(u, x)$ for $x=1,2,3, \ldots$ from the following formulae. For $u=1$, $2,3, \ldots, x-1$

$$
F_{d}(u, x)=\sum_{j=1}^{u} g_{d}(0, j) F_{d}(u-j, x)+\sum_{s=0}^{x-u-1} \sum_{y=u+1}^{\infty} f_{d}(0, s, y)
$$

and for $u=x, x+1, x+2, \ldots$

$$
F_{d}(u, x)=\sum_{j=1}^{u} g_{d}(0, j) F_{d}(u-j, x)
$$

Formula (6.5) follows by considering the first occasion on which the surplus falls below its initial level (if this ever occurs). The first term of (6.4) comes from the same consideration. The second term in (6.4) comes from considering the situation when ruin occurs on the first occasion that the surplus falls below its initial level. In this case the surplus must be no more than $x-u-1$ above its initial level at time $T_{d}-1$ in order for the surplus at that time to be less than $x$. From Gerber (1988, equation (35)), it follows that $f_{d}(0, x, y)=h_{d}(x+y+1) / h_{d}(0)$. Substituting this expression in (6.4) we find that for $u=1,2,3, \ldots, x-1$

$$
F_{d}(u, x)=\sum_{j=1}^{u} g_{d}(0, j) F_{d}(u-j, x)+\sum_{j=u+1}^{x} g_{d}(0, j)
$$

Formulae (6.6) and (6.5), used in this order, provide a stable recursive algorithm for calculating $F_{d}(u, x)$ with the initial value $F_{d}(0, x)$ given by $(6.3)$. We will illustrate the use of this algorithm later in this section.

Let us now consider how to calculate bounds for $F(u, x)$. DICKSON (1992) shows that

$$
F(u, x)=\frac{1-G(0, x)}{1-\psi(0)} \psi(u)-\frac{\psi(0)-G(0, x)}{1-\psi(0)} \text { for } 0 \leq u \leq x
$$

and

$$
F(u, x)=G(u-x, x)-\frac{1-G(0, x)}{1-\psi(0)}(\psi(u-x)-\psi(u)) \text { for } u \geq x
$$

Then for $0 \leq u \leq x$, a lower bound for $F(u, x)$ is $F^{l}(u, x)$, where

$$
F^{\prime}(u, x)=\frac{1-G(0, x)}{1-\psi(0)} \psi^{\prime}(u)-\frac{\psi(0)-G(0, x)}{1-\psi(0)}
$$


and an upper bound is $F^{h}(u, x)$ where

$$
F^{h}(u, x)=\frac{1-G(0, x)}{1-\psi(0)} \psi^{h}(u)-\frac{\psi(0)-G(0, x)}{1-\psi(0)}
$$

For $u \geq x$, a lower bound for $F(u, x)$ is $F^{l}(u, x)$ where

$$
F^{l}(u, x)=G^{l}(u-x, x)-\frac{1-G(0, x)}{1-\psi(0)}\left(\psi^{h}(u-x)-\psi^{l}(u)\right)
$$

and an upper bound is $F^{h}(u, x)$ where

$$
F^{h}(u, x)=G^{h}(u-x, x)-\frac{1-G(0, x)}{1-\psi(0)}\left(\psi^{\prime}(u-x)-\psi^{h}(u)\right)
$$

These bounds are easily calculated by the methods described in Sections 3 and 4 .

Now define

$$
F(u, x, y)=\operatorname{Pr}(T<\infty, U(u, T)>-y \text { and } U(u, \tilde{T})<x)
$$

so that $F(u, x, y)$ gives the (defective) joint distribution of the severity of ruin and the surplus immediately prior to ruin for our basic process. Using the discrete approximation to our basic process, an approximation to $F(u, x, y)$ is $F_{d}^{*}(\beta u, \beta x$, $\beta y)$ where

$$
F_{d}^{*}(u, x, y)=\operatorname{Pr}\left(T_{d}^{*}<\infty, U_{d}\left(u, T_{d}^{*}\right)>-y \text { and } U_{d}\left(u, T_{d}^{*}-1\right)<x\right)
$$

for $u=0,1,2, \ldots, x=1,2,3, \ldots$ and $y=1,2,3, \ldots$. We can compute values of $F_{d}^{*}(u, x, y)$ by first computing values of $F_{d}(u, x, y)$ where

$$
F_{d}(u, x, y)=\operatorname{Pr}\left(T_{d}<\infty, U_{d}\left(u, T_{d}\right) \geq-y \text { and } U_{d}\left(u, T_{d}-1\right)<x\right)
$$

since

$$
F *(u, x, y)=F(u-1, x-1, y)
$$

for $u=1,2,3, \ldots, x=1,2,3, \ldots$ and $y=1,2,3, \ldots$. We can calculate $F_{d}(u, x, y)$ through a stable recursive algorithm. The starting value for the algorithm is

$$
F_{d}(0, x, y)=\sum_{j=0}^{x-1} \sum_{s=1}^{y} f_{d}(0, j, s)
$$

For computational purposes we can write this as

$$
F_{d}(0, x, y)=\frac{1}{h_{d}(0)} \sum_{j=1}^{x}\left(H_{d}(y+j)-H_{d}(j)\right)
$$

An alternative way of writing (6.8) is

$$
F_{d}(0, x, y)=F_{d}(0, x)+G_{d}(0, y)-G_{d}(0, x+y)
$$


which corresponds to the expression for $F(0, x, y)$ for our basic process given by DICKSON and DOS REIS (1994, equation (2.1)).

For $u=1,2,3, \ldots, x-1$ we can use the same reasoning that we used to write formula (6.4) to write

$$
\begin{aligned}
F_{d}(u, x, y) & =\sum_{j=1}^{u} g_{d}(0, j) F_{d}(u-j, x, y)+\sum_{s=0}^{x-u-1} \sum_{j=u+1}^{u+y} f_{d}(0, s, j) \\
& =\sum_{j=1}^{u} g_{d}(0, j) F_{d}(u-j, x, y)+\sum_{j=u+1}^{x}\left(g_{d}(0, j)-g_{d}(0, j+y)\right)
\end{aligned}
$$

Similarly to (6.5), for $u=x, x+1, x+2, \ldots$

$$
F_{d}(u, x, y)=\sum_{j=1}^{u} g_{d}(0, j) F_{d}(u-j, x, y)
$$

Formulae (6.9) and (6.10), with (6.8) as a starting value, give a stable recursive algorithm for calculating $F_{d}(u, x, y)$. An application of this algorithm is given at the end of this section.

Finally, let us consider bounds for $F(u, x, y)$. DiCKsON and DOS REIS (1994) show that

$$
F(u, x, y)=G(u, y)+\frac{\delta(u)}{\delta(0)}(G(0, x)-G(0, x+y)) \text { for } 0 \leq u \leq x
$$

and

$$
\begin{aligned}
F(u, x, y)= & G(u, y)-G(u-x, x+y)+G(u-x, x) \\
& +\frac{\psi(u-x)-\psi(u)}{\delta(0)}(G(0, x)-G(0, x+y)) \text { for } u \geq x
\end{aligned}
$$

Then for $0 \leq u \leq x$, a lower bound for $F(u, x, y)$ is $F^{l}(u, x, y)$, where

$$
F^{l}(u, x, y)=G^{l}(u, y)+\frac{\delta^{h}(u)}{\delta(0)}(G(0, x)-G(0, x+y))
$$

and an upper bound is $F^{h}(u, x, y)$, where

$$
F^{h}(u, x, y)=G^{h}(u, y)+\frac{\delta^{\prime}(u)}{\delta(0)}(G(0, x)-G(0, x+y))
$$

For $u \geq x$, a lower bound for $F(u, x, y)$ is $F^{l}(u, x, y)$, where

$$
\begin{aligned}
F^{\prime}(u, x, y)= & G^{l}(u, y)-G^{h}(u-x, x+y)+G^{l}(u-x, x) \\
& +G(0, x)\left(\psi^{l}(u-x)-\psi^{h}(u)\right) / \delta(0) \\
& +G(0, x+y)\left(\psi^{l}(u)-\psi^{h}(u-x)\right) / \delta(0)
\end{aligned}
$$


and an upper bound is $F^{h}(u, x, y)$, where

$$
\begin{aligned}
F^{h}(u, x, y)= & G^{h}(u, y)-G^{l}(u-x, x+y)+G^{h}(u-x, x) \\
& +G(0, x)\left(\psi^{h}(u-x)-\psi^{l}(u)\right) / \delta(0) \\
& +G(0, x+y)\left(\psi^{h}(u)-\psi^{l}(u-x)\right) / \delta(0)
\end{aligned}
$$

\subsection{Examples}

\subsubsection{Example 7}

Table 6 shows some bounds and approximations to $F(u, x)$ when the individual claim amount distribution is Pareto $(2,1)$, the premium loading factor, $\theta$, is 0.1 and the bounds for $\psi(u)$ and $G(u, y)$ have been calculated as in Sections 3 (using "Method 1") and 4. The key to Table 6 is as follows:

(1) gives the value of $F^{l}(u, x)$,

(2) gives the approximation to $F(u, x)$ calculated from the recursive algorithm for $F_{d}(u, x)$,

(3) gives the approximation to $F(u, x)$ calculated by averaging $F^{l}(u, x)$ and $F^{h}(u, x)$, and

(4) gives the value of $F^{h}(u, x)$.

TABLE 6

See. Example 7, Section 6, for details

\begin{tabular}{lllll}
\hline \hline & & $x=5$ & $x=10$ & $x=15$ \\
\hline$u=10$ & $(1)$ & 0.161668 & 0.287422 & 0.393461 \\
& $(2)$ & 0.169434 & 0.287847 & 0.393936 \\
& $(3)$ & 0.169869 & 0.288154 & 0.394084 \\
& $(4)$ & 0.178070 & 0.288886 & 0.394706 \\
$u=30$ & $(1)$ & 0.064448 & 0.107883 & 0.139130 \\
& $(2)$ & 0.072663 & 0.116525 & 0.148012 \\
& $(3)$ & 0.072851 & 0.116654 & 0.148110 \\
$u=50$ & $(4)$ & 0.081254 & 0.125426 & 0.157089 \\
& $(1)$ & 0.035739 & 0.060270 & 0.077271 \\
& $(2)$ & 0.042324 & 0.067322 & 0.084765 \\
& $(3)$ & 0.042434 & 0.067398 & 0.084823 \\
& $(4)$ & 0.049130 & 0.074526 & 0.092375 \\
\hline
\end{tabular}

\subsubsection{Example 8}

Table 7 shows some bounds, approximations and exact values for $F(u, x, y)$ when the individual claim amount distribution is exponential with mean 1 , the premium loading factor, $\theta$, is 0.1 and the bounds for $\psi(u)$ and $G(u, y)$ have been calculated as in Sections 3 (using "Method 1") and 4. The key to Table 7 is as follows:

(1) gives the value of $F^{l}(u, x, y)$,

(2) gives the exact value of $F(u, x, y)$,

(3) gives the approximation to $F(u, x, y)$ calculated from the recursive algorithm for $F_{d}(u, x, y)$, 
(4) gives the approximation to $F(u, x, y)$, calculated by averaging $F^{l}(u, x, y)$ and $F^{h}(u, x, y)$, and

(5) gives the value of $F^{h}(u, x, y)$.

TABLE 7

See Example 8, Section 6, For Detalls

\begin{tabular}{|c|c|c|c|c|}
\hline & & $x=y=1$ & $x=y=3$ & $x=y=5$ \\
\hline$u=20$ & $\begin{array}{l}(1) \\
(2) \\
(3) \\
(4) \\
(5)\end{array}$ & $\begin{array}{r}-0.070275 \\
0.023040 \\
0.022529 \\
0.023041 \\
0.116357\end{array}$ & $\begin{array}{r}-0.021141 \\
0.109159 \\
0.108601 \\
0.109161 \\
0.239464\end{array}$ & $\begin{array}{l}0.000549 \\
0.139331 \\
0.139137 \\
0.139333 \\
0.278116\end{array}$ \\
\hline$u=60$ & $\begin{array}{l}(1) \\
(2) \\
(3) \\
(4) \\
(5)\end{array}$ & $\begin{array}{r}-0.006932 \\
0.000607 \\
0.000594 \\
0.000607 \\
0.008147\end{array}$ & $\begin{array}{r}-0.008188 \\
0.002876 \\
0.002862 \\
0.002877 \\
0.013942\end{array}$ & $\begin{array}{r}-0.008862 \\
0.003671 \\
0.003666 \\
0.003672 \\
0.016206\end{array}$ \\
\hline$u=100$ & $\begin{array}{l}(1) \\
(2) \\
(3) \\
(4) \\
(5)\end{array}$ & $\begin{array}{r}-0.000317 \\
0.000016 \\
0.000016 \\
0.000016 \\
0.000349\end{array}$ & $\begin{array}{r}-0.000417 \\
0.000076 \\
0.000075 \\
0.000076 \\
0.000569\end{array}$ & $\begin{array}{r}-0.000467 \\
0.000097 \\
0.000097 \\
0.000097 \\
0.000661\end{array}$ \\
\hline
\end{tabular}

\subsubsection{Comments on Examples 7 and 8}

(i) In each case, the approximations are close together, and we can see from Example 8 that the approximations are close to the true values. As with the approximations to $G(u, y)$ when the individual claim amount distribution is exponential, approximations to $F(u, x, y)$ based on the bounds are slightly better for small values of $u$.

(ii) Example 8 illustrates that the lower bound for $F(u, x, y)$ can be negative, as can be the lower bound for $F(u, x)$. Thus, the bounds themselves may be of little practical value. However, averaging the bounds produces reasonable approximations since this process simply averages bounds for the functions $\psi(\cdot)$ and $G(\cdot, \cdot)$, and the bounds for $F(u, x)$ and $F(u, x, y)$ depend on the bounds for these functions.

(iii) We have seen in Example 1 that averaging bounds for $\psi(u)$ gives an excellent approximation to $\psi(u)$. Hence, when $u \leq x$ the average of the bounds for $F(u, x)$ should be a very good approximation to $F(u, x)$ since these bounds are linear functions of the bounds on $\psi(u)$.

\section{REFERENCES}

De Vylder, F. and Goovaerts, M.J. (1988) Recursive calculation of finite time ruin probabilities. Insurance: Mathematics and Economics, 7, 1-8.

Dickson, D.C.M. (1992) On the distribution of the surplus prior to ruin. Insurance: Mathematics and Economics, 11, 191-207.

DICKSON, D.C.M. (1994) Some comments on the compound binomial model. ASTIN Bulletin, 24, 33-45. 
DiCkSON, D.C. M. and Dos REIS, A.E. (1994) Ruin problems and dual events. Insurance: Mathematics and Economics, 14, 51-60.

DiCKSON, D.C.M. and WATERS, H.R. (1991) Recursive calculation of survival probabilities. ASTIN Bulletin, 21, 199-221.

Dickson, D.C. M. and WATERS, H.R. (1992) The probability and severity of ruin in finite and in infinite time. ASTIN Bulletin, 22, 177-190

Dos REls, A. E. (1993) How long is the surplus below zero? Insurance: Mathematics and Economics, 12 , 23-38.

Dufresne, F. and Gerber, H.U. (1989) Three methods to calculate the probability of ruin. ASTIN Bulletin, 19, 71-90.

Gerber, H.U. (1988) Mathematical fun with the compound binomial process. ASTIN Bulletin, 18, $161-168$.

Gerber, H. U., Goovaerts, M.J. and KaAs, R. (1987) On the probability and severity of ruin. ASTIN Bulletin, 17, 151-163.

Goovaerts, M.J. and DE VYLDER, F. (1984) A stable recursive algorithm for evaluation of ultimate ruin probabilities. ASTIN Bulletin, 14, 53-59.

PANJER, H. H. (1981) Recursive calculation of a family of compound distributions. ASTIN Bulletin, 12, 22-26.

PANJER, H.H. (1986) Direct calculation of ruin probabilities. Journal of Risk and Insurance, 53, $521-529$.

Panjer, H. H. and Lutek, B. (1983) Practical aspects of stop-loss calculations. Insurance : Mathematics and Economics, 2, 159-177.

PANJER, H.H. and WANG, S. (1993) On the stability of recursive formulas. ASTIN Bulletin, 23, $227-258$

Panjer, H.H. and Willmot, G. E. (1992) Insurance Risk Models. Society of Actuaries, Schaumberg, IL.

SHIU, E.S.W. (1989) The probability of eventual ruin in the compound binomial model. ASTIN Bulletin, 19. $179-190$.

WILLMOT, G.E. (1992) Ruin probabilities in the compound binomial model. Insurance: Mathematics and Economics, 12, 133-142.

DAvid C. M. DiCKSON

Centre for Actuarial Studies, Faculty of Economics and Commerce

University of Melbourne

Parkville, Victoria 3052, Australia

Alfredo D. Egídio dos Reis

Departamento de Matemática, Instituto Superior de Economia e Gestão

Rua Miguel Lupi 20, P-1200 Lisbon, Portugal

HOWARD R. WATERS

Department of Actuarial Mathematics and Statistics

Heriot-Watt University, Edinburgh

Midlothian EHI4 4AS, Great Britain 